\title{
Impedance spectroscopy of perovskite/ contact interface: Beneficial chemical reactivity effect
}

Cite as: J. Chem. Phys. 151, 124201 (2019); https://doi.org/10.1063/1.5111925

Submitted: 01 June 2019 . Accepted: 05 September 2019 . Published Online: 25 September 2019

Clara Aranda (D), Juan Bisquert (D), and Antonio Guerrero (D)

\section{COLLECTIONS}

Paper published as part of the special topic on Lead Halide Perovskites

Note: The paper is part of the JCP Special Topic on Lead Halide Perovskites.
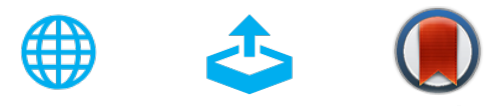

\section{ARTICLES YOU MAY BE INTERESTED IN}

Morphotropic phase boundary-like properties in a ferroelectric-paraelectric nanocomposite Journal of Applied Physics 126, 124102 (2019); https:// doi.org/10.1063/1.5113623

Crystallization kinetics and role of stress in Al induced layer exchange crystallization process of amorphous SiGe thin film on glass

Journal of Applied Physics 126, 125303 (2019); https://doi.org/10.1063/1.5115539

Role of device architecture and $\mathrm{AlO}_{X}$ interlayer in organic Schottky diodes and their interpretation by analytical modeling

Journal of Applied Physics 126, 125501 (2019); https://doi.org/10.1063/1.5109083

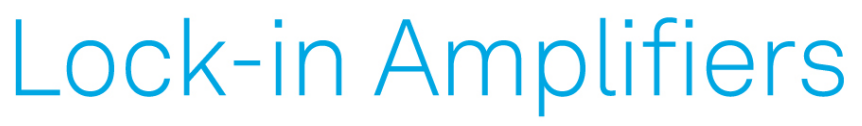

... and more, from DC to $600 \mathrm{MHz}$ Watch 


\title{
Impedance spectroscopy of perovskite/contact interface: Beneficial chemical reactivity effect
}

Cite as: J. Chem. Phys. 151, 124201 (2019); doi: 10.1063/1.5111925

Submitted: 1 June 2019 • Accepted: 5 September 2019 •

Published Online: 25 September 2019

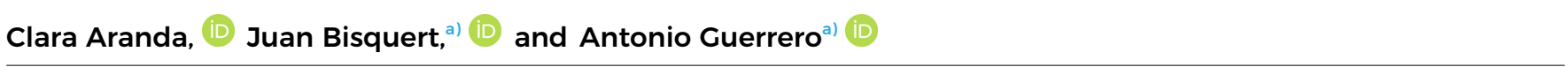

\author{
AFFILIATIONS \\ Institute of Advanced Materials (INAM), Universitat Jaume I, 12006 Castelló, Spain
}

Note: The paper is part of the JCP Special Topic on Lead Halide Perovskites.

a) Electronic addresses: bisquert@uji.es and aguerrer@uji.es

\begin{abstract}
Understanding chemical reactivity of lead halide perovskite materials with contacts is crucial to improve the stability of these optoelectronic devices. The study of the physical and chemical interactions at the interfacial region is still one of the most challenging tasks in this field. We investigate a configuration based on the direct contact of gold ( $\mathrm{Au}$ ) with highly crystalline methylammonium lead bromide perovskite $\left(\mathrm{MAPbBr}_{3}\right)$, in comparison with the presence of an organic interlayer. The metal contact clearly shows the double layer capacitance that can be monitored by Impedance Spectroscopy (IS). Measurements in the dark reveal the frequencies where a reduction in charge accumulation occurs, related to ionic reactivity with the external contacts. Under light, this chemical reaction is favored and the newly formed contact improves the performance of the solar cell. The IS results show that reactivity proceeds at timescales longer than $100 \mathrm{~s}$, reducing the recombination kinetics under 1 sun illumination conditions, increasing the photovoltage and photocurrent that can be extracted. This work presents IS as a nondestructive in operando tool to monitor the kinetics of the ionic double layer formation and the reactivity of methylammonium bromide perovskite material with contacts decoupling as well this information from other resistive and capacitive contributions.
\end{abstract}

Published under license by AIP Publishing. https://doi.org/10.1063/1.5111925

\section{INTRODUCTION}

Photovoltaic devices based on lead halide perovskites have emerged as strong candidates to compete with commercial technologies with a steady increase in efficiencies (i.e., Power Conversion Efficiencies), currently with certified and published values of 23.3\%. ${ }^{1}$ In addition, lead halide perovskites find multiple applications such as in Light Emitting Diodes, photodetectors, or X-ray detectors. $^{2-4}$ One common feature for all these optoelectronic devices is the necessity to use external contacts capable to either inject or extract current. The variety of materials used to such end is vast and it is tailor-made for the specific application. Despite the impressive achievements, stability of devices still remains one of the biggest challenges, where the perovskite/external interface reactivity is clearly at the heart of the problem.

Monitoring interfacial reactivity in optoelectronic devices represents a great difficulty since the reaction is confined to a few nanometers width at the proximity of the contact being enough to modify severely the electronic properties of the absorber material. The interface is buried inside the complete device and analysis of a half stack is not enough to satisfy the operating conditions promoting the chemical reaction. Chemical spatially resolved techniques to detect reaction products require ion milling, which is a destructive technique. ${ }^{7,8}$ As an extra disadvantage, the concentration of reaction products at the interface is too small, restricting the analysis to element distribution with a high degree of uncertainty, impeding as well kinetic studies in operating devices. Alternatively, electrical techniques do not offer chemical information but can provide data about the reaction kinetics.

Impedance spectroscopy (IS) represents a very useful technique to probe the kinetics of physical and chemical processes in semiconductor materials and batteries. ${ }^{9,10}$ Resistive and capacitive contributions are separated by IS according to the kinetics of the processes. The technique has been applied with relative success in perovskite solar cells field due to the great variety of effects occurring simultaneously, but the potential to unveil chemical and physical 
processes in operando is very attractive. ${ }^{11,12}$ The major disadvantages of the technique is that (i) the interpretation of the data is not direct nor straightforward and (ii) the technique is totally blind to the type of chemistry/interaction taking place at the interface. To overcome these issues, we have designed several experiments in the past to build information that allows the interpretation of the data. For example, by variation of the thickness of $\mathrm{MAPbBr}_{3}$ monocrystals, we have been able to separate electronic and ionic current to identify ionic migration in the bulk. ${ }^{13}$ In these experiments, conditions were identified and selected to avoid electronic current: minimized doping of the perovskite layer and measurement in the dark, close to equilibrium conditions with applied voltages near to $0 \mathrm{~V}$. Similarly, previous analyses of IS data in the perovskite solar cell have indicated a different response of the central perovskite layer and the interfaces with contacts. ${ }^{11}$ Very recently, Au interaction with halide perovskites to form oxidized gold halide species has shown to improve the charge injection/extraction and the present report benefits from this knowledge. ${ }^{14}$ However, the kinetics of the chemical reactions between perovskite and contacts have not been previously analyzed by IS.

In this work, solar cells consisting on highly crystalline $\mathrm{MAPbBr}_{3} / \mathrm{Au}$ interface, in contraposition to cells that do contain Spiro-OMeTAD as hole conductor layer in the standard configuration, allow to clearly identify the interfacial effects by monitoring the changes observed during photovoltaic operation. The cell with direct $\mathrm{MAPbBr} / \mathrm{Au}$ interface readily forms oxidized gold halide species, as previously reported, and reveals the kinetics of a progressive activation of the solar cell performance at a time scale that can be proved by IS. By comparing IS in dark and under illumination, we obtain a picture of the changes at the contact that indicates the ionic reactivity process in combination with other capacitive and electronic contributions.

\section{RESULTS AND DISCUSSION}

In order to obtain a model system to probe the reactivity of perovskite-metal interface, solar cells have been fabricated in regular configuration: $\mathrm{FTO} / \mathrm{c}-\mathrm{TiO}_{2} / \mathrm{m}-\mathrm{TiO}_{2} / \mathrm{MAPbBr}_{3} / \mathrm{Au}(\mathrm{MA}=$ methylammonium). We have previously reported a method to produce high quality $\mathrm{MAPbBr}_{3}$ films of nearly $1 \mu \mathrm{m}$ free of pinholes. ${ }^{1 .}$ In this work, similar devices are prepared; see the cross-sectional SEM image of this $\mathrm{MAPbBr}_{3}$ device [Fig. 1(a)] that shows distinctively uniform, voids-free feature of the perovskite layer. We reported previously that the highly crystalline material in combination with further tuning of the electron transport layer provided photovoltages approaching $1.6 \mathrm{~V}$ and power conversion efficiencies of $6.6 \%$.

In the absence of Spiro-OMeTAD, the current-voltage $J-V$ curve undergoes a significant change during the first cycles of measurement of the device [Fig. 1(b)]. Note that fast scan rates of 500 $\mathrm{mV} / \mathrm{s}$ have been used to avoid modification of the external interfaces during the $J-V$ measurement. In Fig. 1(b) is shown a representative $J$ - $V$ curve of a fresh no Hole Selective Layer (HSL) device and how subsequent fast cycling under illumination improves the performance of the solar cell. This photovoltaic behavior allows us to ascribe the improvement of the solar cell performance to a chemical modification at the perovskite/Au interface. This type of
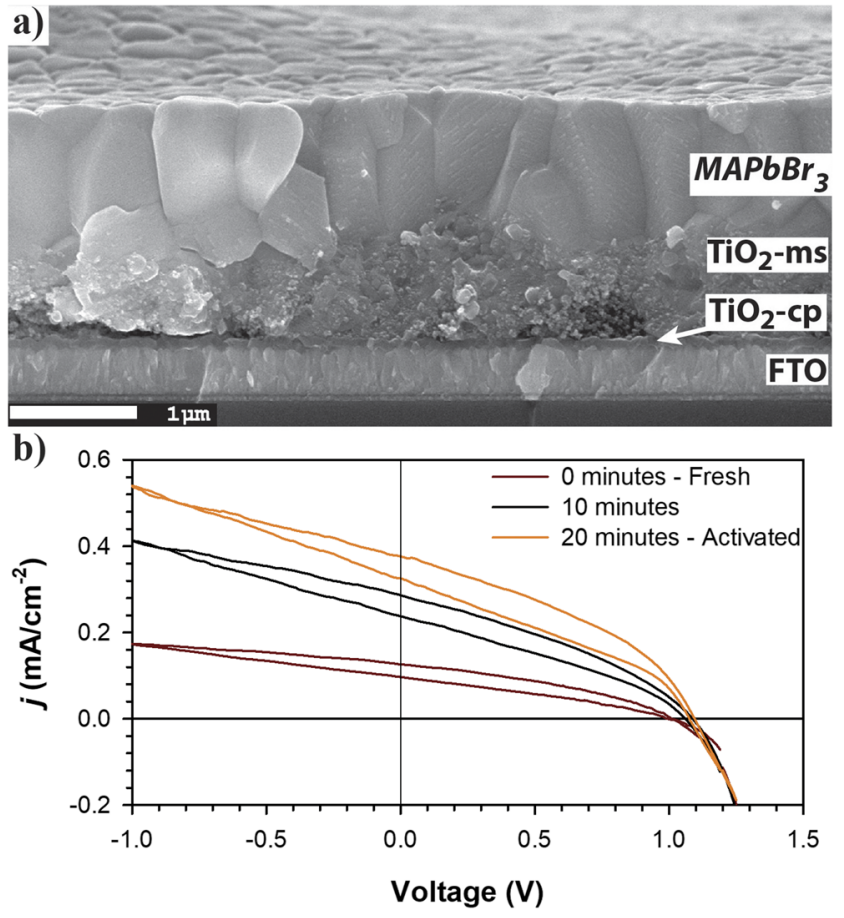

FIG. 1. (a) Cross-sectional SEM image of a photovoltaic device containing highly crystalline $\mathrm{MAPbBr}_{3}$. (b) Representative $\mathrm{J}-\mathrm{V}$ curves of a fresh device and the response after cycling of the device under illumination during increasing times. Scan rate of $500 \mathrm{mV} / \mathrm{s}$.

improvement has also been observed in a symmetric configuration with monocrystalline $\mathrm{MAPbBr}_{3}$ in the absence of $\mathrm{TiO}_{2}$ and evaporated gold at both contacts. ${ }^{14}$ In the previous work, improved charge extraction/injection was observed when oxidized gold halide species are formed under the presence of an electrical field. Therefore, here we denote activated samples those that contain such oxidized gold species at the interface. To further confirm that gold halide species are responsible for the improved performance, control devices containing Spiro-OMeTAD do not show such large variations in $J-V$ curves as a function of the scan rates (see the supplementary material). To understand the kinetics of this chemical reactivity and the electrical consequences of this interfacial modification, the impedance response is in depth investigated. In the following text, we will denote as "fresh" and "activated" samples that correspond to $J$ - $V$ curves as those presented in Fig. 1(b). The precise sequence of the measurements is important as described in the experimental section.

During IS measurement, a DC voltage is applied to the sample either under light or dark conditions and a small AC voltage perturbation is superimposed with a wide range of frequencies. The measurement sweeps frequencies starting from high to low frequencies, with high frequency (HF) data points obtained close to the origin of $Z^{\prime}$ and $-Z^{\prime \prime}$. Depending on the lowest frequency limit, the measurement typically takes about 2-15 min for one DC voltage. A useful method to represent the impedance data is the complex impedance plot, where $\mathrm{x}$-axis quantity $Z^{\prime}=\operatorname{Re}(Z)$ is related to resistive elements 
and the $y$-axis quantity $Z^{\prime \prime}=-\operatorname{Im}(Z)$ to capacitive elements. A resistive element connected in parallel to a capacitor (RC) leads to an arc in the complex impedance plot. In perovskite research for devices containing Spiro-OMeTAD, the most repeated feature in the dark is the observation of two arcs. ${ }^{11,12}$

Here, devices containing $\mathrm{MAPbBr}_{3}$ were studied by IS in the dark and they are compared with a reference device containing Spiro-OMeTAD [Figs. 2(a)-2(c)], delivering efficiencies as previously reported approaching $7 \%$. For measurements in the dark, a fresh device is measured at $0 \mathrm{~V}$, again at $0.2 \mathrm{~V}$ (before activation) and the same device is measured at $0 \mathrm{~V}$ after activation during cycling under illumination, as described in the experimental section.

In the dark, devices without HSL display two arcs in the complex impedance plots while the device containing Spiro-OMeTAD shows a spectrum that resembles three arcs (see the supplementary material). The high frequency (HF) arc is observed at frequencies $>1 \mathrm{kHz}$ as shown in Figs. 2(a) and 2(b), and it contains information about carrier transport in the perovskite layer and the resistivity of the contacts. ${ }^{11}$ As the resistivity of Spiro-OMeTAD is higher than that of gold, this HF resistance is higher. The fresh device measured at $0 \mathrm{~V}$ totally overlaps with the device measured at $0.2 \mathrm{~V}$, and the HF resistance of the activated device is higher than those for fresh devices. The nature of the low frequency (LF) arc measured in the dark is not so well understood and rather than a simple resistance, this arc may contain the combination of two separate resistances, which are mixed in IS response but can be clearly separated using Intensity Modulated Photocurrent Spectroscopy (IMPS). ${ }^{16}$ Only in a few cases the nature of these separate leading to clearly differentiated intermediate frequency (IF) and LF resistances. For monocrystalline $\mathrm{MAPbBr}_{3}$ perovskite material, the IF resistance displays a vertical line with pure capacitive contribution. ${ }^{13}$ However, Fig. 2(c) shows an arc at $\mathrm{LF}(<0.1 \mathrm{~Hz})$ that begins to close, indicating a certain amount of charge transfer at the contact, which may be due either to electron transfer or ionic reactivity. ${ }^{13}$ Here, we note that our polycrystalline material is highly crystalline as observed by SEM images and previously reported, so that grain boundary effects can be excluded to account for these observations.

Under illumination and open circuit $\left(V_{o c}\right)$ conditions, samples not containing Spiro-OMeTAD also show two arcs in the complex impedance plots. It is also observed the effect of a noisy signal with inductive elements that crosses the y-axis to negative values in the low frequency region [Fig. 2(e)]. As the perovskite is a photoconductive material and the conductivity increases under illumination by many orders of magnitude, the resistance related to the bulk transport is usually not observed by IS. In this case, resistances correspond to losses mainly from recombination processes, both in the bulk perovskite and at the interfaces. ${ }^{19}$ Large values of these resistances denote high resistance toward the recombination of carriers and correlate with a better performance of the device. ${ }^{15}$ Here, we note a higher HF resistance for the activated sample, as expected for the best performance of the activated sample, but similar LF resistance for both samples. In addition, we highlight that the very low frequency region shows noisy behavior, and an ionic component of LF resistance is not discarded as it will be described next.
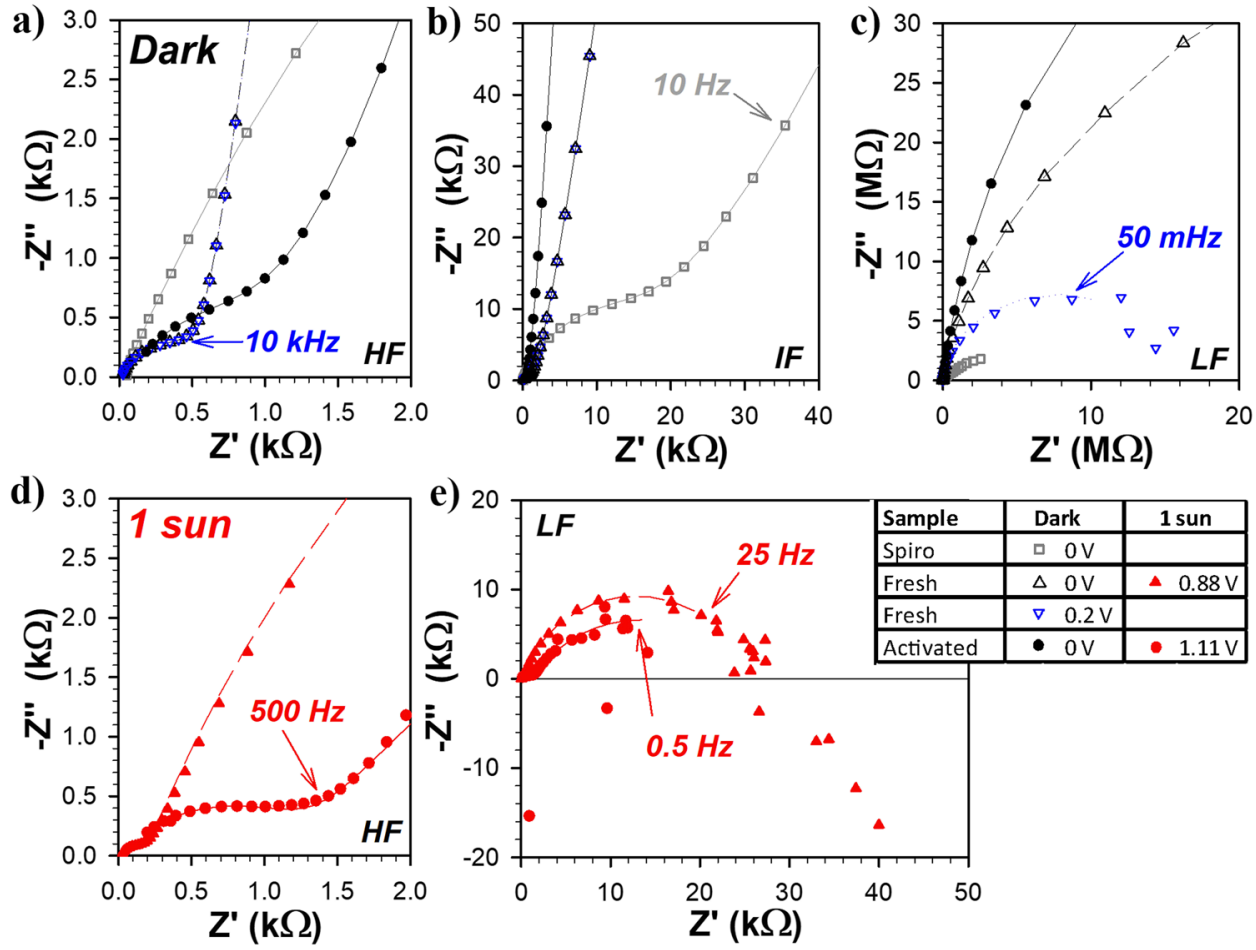

FIG. 2. Impedance spectroscopy results of devices measured in the dark under different conditions, as indicated. Complex impedance plot of spectra in dark showing different frequency regions: (a) High Frequency (HF) (>1 kHz), (b) Intermediate frequency (IF) $(>0.1 \mathrm{~Hz})$, and (c) Low frequency (LF) $(>0.01 \mathrm{~Hz})$. [(d) and (e)] Spectra recorded under illumination at 1 sun light intensity for fresh and activated devices, which do not contain Spiro-OMeTAD. 
The understanding of the nature of capacitive behaviors is crucial to explain operation mechanisms related to charge distribution in solar cells. A different way to visualize the same data as that shown in Fig. 2 is by plotting the capacitance as a function of the frequency (Fig. 3). We observe that the evolution of the capacitance vs frequency shows three different contributions as indicated by three separated plateaus. Normally, two capacitances are observed by using IS, attributed to bulk and contact polarization. However, sometimes a third capacitive contribution is observed in the IF domain when devices are measured by IS. ${ }^{11,16,17}$ In this case, one may expect that the capacitive contributions of the two contacts can be separately observed.

In order to obtain an interpretation of the capacitances, we note first the plateau at HF that is attributed to the bulk dielectric polarization of the perovskite. ${ }^{10,15-18}$ Thus, the HF capacitance is a geometrical capacitance, $C=\varepsilon \varepsilon_{0} / d$, where $\varepsilon$ is the relative dielectric constant of the perovskite material, $\varepsilon_{0}$ is the vacuum dielectric constant, and $d$ is the thickness of the perovskite film. For a perovskite thickness of $800 \mathrm{~nm}$ and capacitance values of approximately $4 \times 10^{-8} \mathrm{~F} \mathrm{~cm}^{-2}$, dielectric constant of the material of 35 is calculated. All devices, including the device containing Spiro-OMeTAD, tend to these capacitance values, including under illumination conditions.

The IF and LF regions measured in the dark show an increase of capacitance related to formation of a double layer at the contacts, with values of the order $10^{-6} \mathrm{~F} \mathrm{~cm}^{-2}$, typically associated with the Helmholtz ionic layer at the perovskite contact surface, marked as a flat line in the plot. ${ }^{19,20}$ Here, it is important to highlight the different behavior of the samples with $\mathrm{Au}$ and Spiro-OMeTAD. For the latter, there is a continuous rise of capacitance up to very low frequencies. This is the characteristic response of perovskite solar cells, observed in many reported measurements. ${ }^{21}$ In contrast, for the Au contact without Spiro-OMeTAD, the capacitance is nearly flat. As remarked before, there are three main capacitances in the system: bulk, perovskite/ $\mathrm{TiO}_{2}$, and perovskite/(Au or organic). In the cell with Spiro-OMeTAD, the steady increase in the low frequency capacitance is a combination of the two contact capacitances. However, when we eliminate the Spiro-OMeTAD, we remove a large double layer capacitance from this interface and the remaining capacitance is lower as shown in the simulations below. Here, it is important to observe that in the literature generation of the ionic double layer has normally been ascribed to the $\mathrm{TiO}_{2} /$ perovskite interface, based on the suppression of the large capacitance in the inverted cell with PCBM contact. ${ }^{22}$ Our new observation indicates that in usual measurements, the LF capacitance measured in the dark is a combination of the two contacts and the contact resistances will play a very important role. Recently, the charge stored at both contacts has been observed by Kelvin probe force microscopy and in our work we can quantify it by electrical methods. ${ }^{23}$ This remark is important for the observations under illumination, and it is in agreement for changes of surface polarization observed with modification of organic conductor hole extraction layers. ${ }^{24}$ As an additional feature, the activated sample does not show the intermediate capacitive process related to ionic double layer generation, going directly to the LF response. We assume that the reason for this is that ions have reacted with the gold electrode during the activation process and are no longer available, at the same time reducing the contact resistance.

The fresh device measured before activation in the dark at $0.2 \mathrm{~V}$ shows the same values of impedance $Z$ measured at $0 \mathrm{~V}$. The spectra in Fig. 2 practically overlap, which confirms the high reproducibility of the $\mathrm{MAPbBr}_{3}$ solar cells presented in this paper. However, in

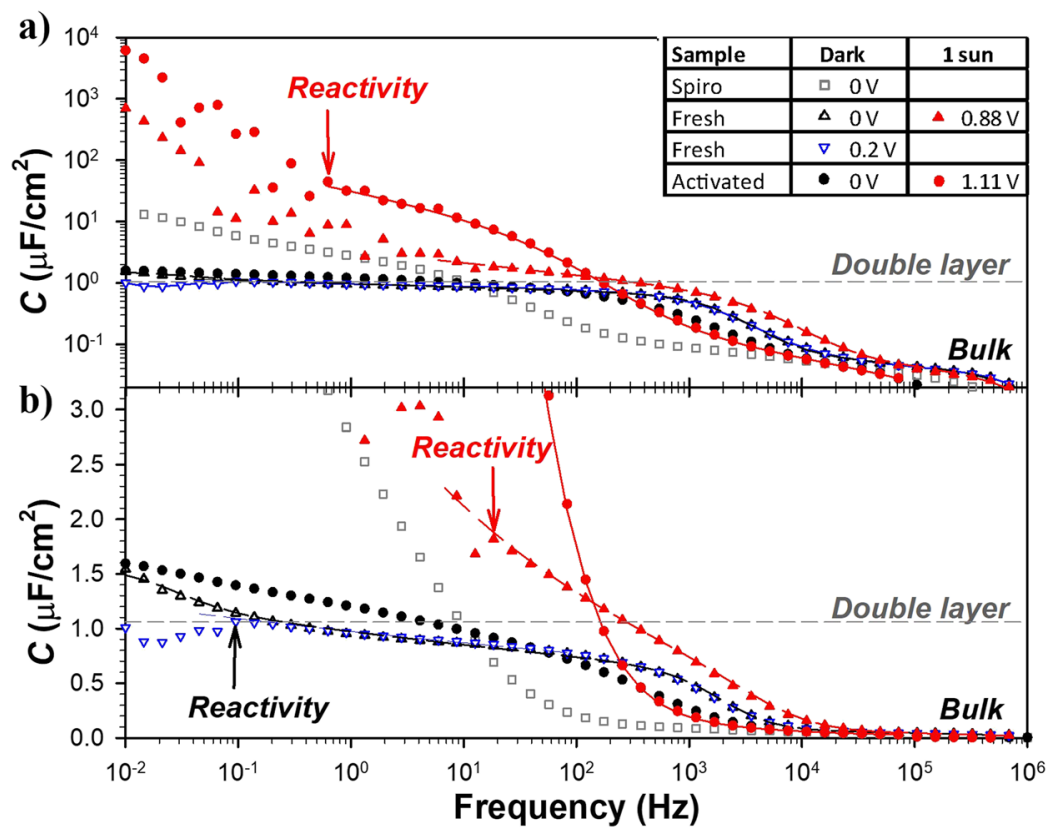

FIG. 3. Impedance spectroscopy results of devices measured in the dark under different conditions. Capacitance vs frequency plot showing different vertical scales: (a) logarithmic and (b) linear plot around the value of the Helmholtz capacitance, indicated with a straight baseline. 
the domain of very low frequencies, an unexpected decrease in the LF capacitance response is obtained [Fig. 3(b)]. Since devices containing Spiro-OMeTAD do not show this behavior, we attribute this drop to a chemical reaction between $\mathrm{Au}$ and the ions to form oxidized gold halide species that reduces the contact resistance, which may involve inductive elements connected to negative capacitance as reported before. ${ }^{10,14}$ This type of chemical reaction leads to very noisy signal as noted in Fig. 2(c).

Under illumination conditions near 1 sun, a different behavior is expected. As observed many times in the literature, a very large excess of capacitance is generated at low frequency, over the Helmholtz ionic layer, often associated with an electronic accumulation capacitance at the perovskite $/ \mathrm{TiO}_{2}$ interface. ${ }^{25}$ However, significant differences are observed between the fresh and activated samples in Fig. 3(a). At IF $(10 \mathrm{~Hz})$, the former barely overcomes the value of the double layer, which indicates that the total capacitance is still pinned at the Helmholtz layer of the fresh $\mathrm{MAPbBr}_{3} / \mathrm{Au}$ contact. In contrast to this, the activated sample allows a continuous growth of the capacitance towards low frequencies. We attribute this difference to the construction of an halide-gold layer, with $\mathrm{Au}$ at the surface being oxidized, which improves the contact properties and is responsible for the incremental photocurrent recorded in Fig. 1. ${ }^{14}$ The modification of the contact caused by ionic reactivity during $J$ - $V$ cycling is outlined in Fig. 4. The nature of the species generated is not the focus of this work and has been studied elsewhere.

Under illumination and at very low frequencies, around $0.1 \mathrm{~Hz}$, the capacitance continues to increase [Fig. 3(a)] for both fresh and activated samples, coinciding with the inductive effect and negative capacitance. This type of inductive element has been previously correlated with electrochemical reactions and corrosion in several materials. ${ }^{26,27}$ We assume that the LF capacitive rise under illumination conditions in the LF region still represents an electronic accumulation at the interfaces, but the noisy behavior observed in this region, already remarked in the measurements in the dark, may suggest an effect due to the ionic reactivity. For the fresh sample, the noise begins at frequencies of $\sim 10 \mathrm{~Hz}$ related to a steady supply of ions, and under illumination conditions, the chemical reactivity is favored. Alternatively, the activated sample only shows the noise at frequencies below $\sim 0.5 \mathrm{~Hz}$, indicating a more costly generation of the ionic double layer and reactivity, since now the

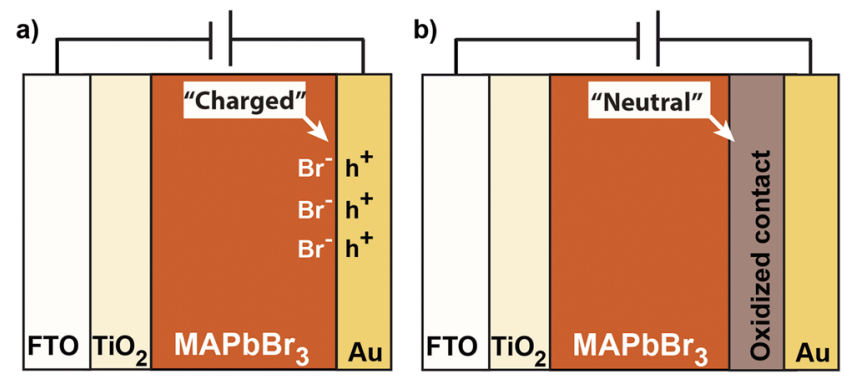

FIG. 4. Diagrams representing the interface modification that leads to an increase of intermediate frequency capacitance and enhanced photocurrent. (a) Pure ionic double layer capacitance observed at intermediate frequencies and (b) intermediate oxidized layer allows surface accumulation of electronic carriers and enhances interfacial charge transfer. supply of ions to the metal contact is less effective. The frequency at which noise appears is then very revealing and indicates the timescale at which ions arriving at the external interface begin to react with the contact. ${ }^{6}$ Here, we highlight that devices containing Spiro-OMeTAD occasionally also show the noisy behavior, and in these cases, we attribute the noise to partial coverage of the perovskite by Spiro-OMeTAD that enable areas of direct contact perovskite/Au.

We can summarize the previous interpretation in an equivalent circuit shown in Fig. 5(a), which allows us to describe the impedance spectra measured with 2 arcs and three capacitive processes. The circuit is composed by the bulk geometric capacitance connected with the HF resistance $\left(R_{3}\right)$ that arises from conductivity of perovskite and recombination processes. In addition, two separate surface capacitances with the corresponding resistive elements (one $R C$ ) arise for each interface of the perovskite with the external contacts. As noted above, the couplings $R_{1} C_{1}$ and $R_{2} C_{2}$ lead to a single arc and two capacitive processes. In practice, both $R C$ related to the contacts can appear either in the IF or LF in the complex plane impedance spectra depending on the individual elements as they have a large impact in the overall spectra. To show this effect, we have carried out simulations using parameters close to those that fit the fresh spectra under dark conditions $\left(R_{\text {series }}=20 \Omega, C_{\text {bulk }}=2\right.$ $\times 10^{-8} \mathrm{~F}$ and $R_{1}=2.8 \times 10^{5} \Omega, C_{1}=2 \times 10^{-6} \mathrm{~F}$ ) and the results are shown in Fig. 5. A reduction in the HF resistance $\left(R_{3}\right)$ [Fig. 5(b)] displaces the onset of the increase in the IF and LF capacitances to higher frequencies. This is an effect of the decrease in the characteristic time $\tau$ arising from the product $R_{3} \times C_{\text {bulk }}$. In Fig. 2(a), a slower onset has been observed in the activated sample measured in both the dark and under illumination in comparison to the fresh sample. The reason is that ions are driven to the contacts and the doping in the bulk of the perovskite material decreases as reported previously. ${ }^{28}$ Similarly, when the device is illuminated, the increased conductivity of the perovskite layer, as it is photoconductive material, leads to a decrease in resistance and the onset in capacitance is shifted toward higher frequencies. The effect of the capacitance in hysteresis and fill factor of perovskite solar cells has been remarked elsewhere. ${ }^{16}$ Alternatively, a change in one of the contact resistance $\mathrm{R}_{2}$ [Fig. 5(c)] will modify the achievable capacitance in IF and LF but has little effect on the onset, which will determine the kinetics of the hysteresis phenomena. This could be the case of a device containing Spiro-OMeTAD that shows a spectrum that resembles three arcs; in this case, a large IF resistance allows the observation of higher LF capacitances in the order of tens of $\mu \mathrm{F} / \mathrm{cm}^{2}$. Finally, a change in one of the contact capacitances will modify both IF and LF plateau [Fig. 5(d)]. It needs to be highlighted that the spectrum is very sensitive to the capacitance measured at the interfaces where small modifications in the capacitance lead to large differences in the observation of two or three arcs. As observed in the previous data on the combination of RC in series, where both resistance and capacitance are connected, the spectrum is often not very intuitive and fitting of the results is required to establish the values of $R$ and $C$. The results of the fitting of data to the equivalent circuit model are presented in the supplementary material.

Regarding the equivalent circuit needed to fit adequately data measured under illumination conditions, this necessarily needs to be more complex as the effect of an inductance is usually observed. Here, we have not included this element due to the very noisy 

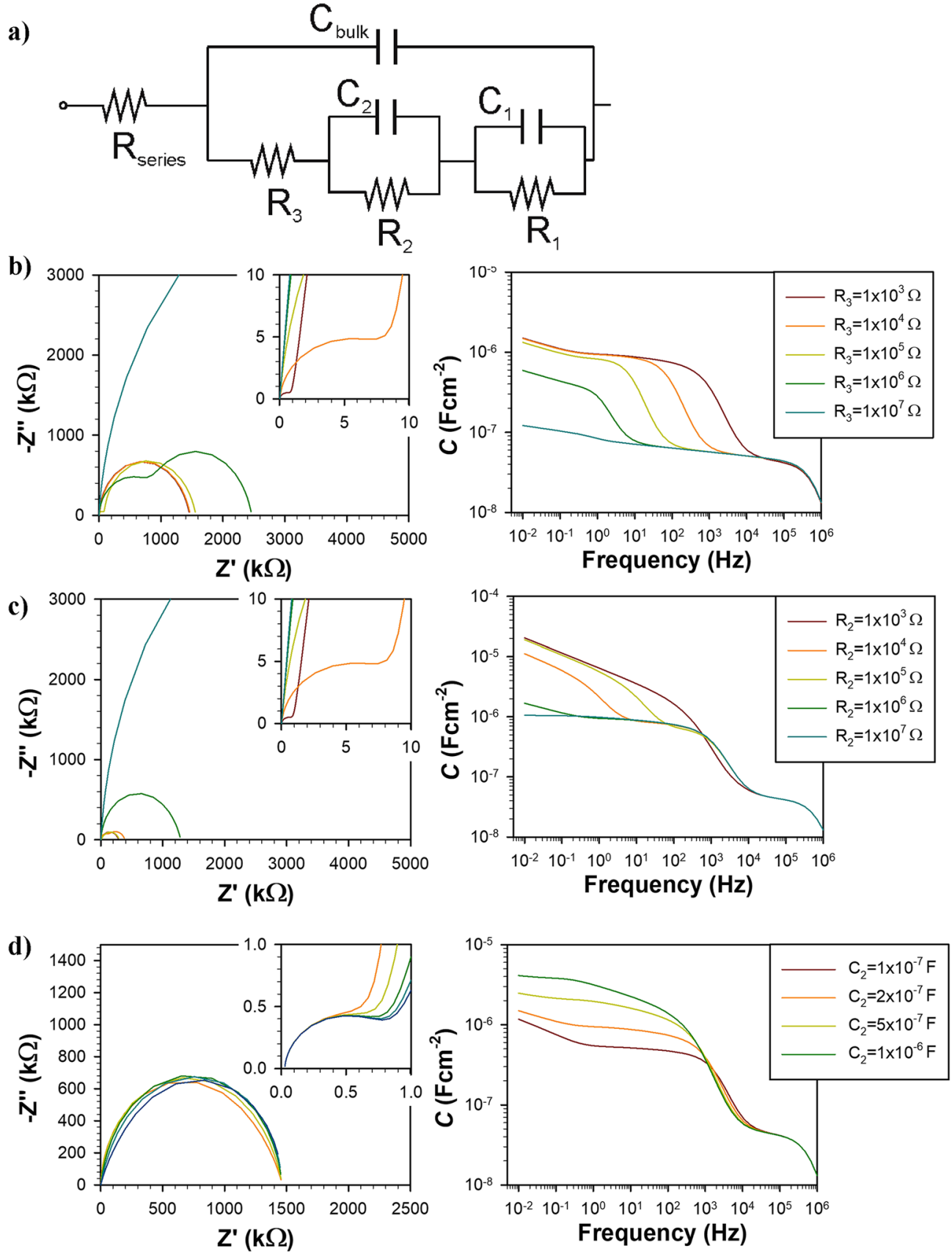

FIG. 5. (a) Equivalent circuit used to fit the IS data measured in the dark. [(b)-(d)] Simulation spectra using the equivalent circuit in (a) to understand the effect of IF features Common parameters: $R_{\text {series }}=20 \Omega, C_{\text {bulk }}=2 \times 10^{-8} \mathrm{~F}, R_{1}=2.8 \times 10^{5} \Omega$, and $C_{1}=2 \times 10^{-6} \mathrm{~F}$. (b) Modification of $R_{3}$ with fixed $R_{2}=1 \times 10^{6} \Omega$ and $C_{2}=2 \times 10^{-7} \mathrm{~F}$. (c) Modification of $R_{2}$ with fixed $R_{3}=1000 \Omega$ and $C_{2}=2 \times 10^{-7} \mathrm{~F}$. (d) Modification of $C_{2}$ with fixed $R_{3}=1000 \Omega$ and $R_{2}=1 \times 10^{6} \Omega$. CPE elements have been used for the contact capacitances with exponential factors of 0.8 . In the graph, capacitance has been normalized using an active area of $0.2 \mathrm{~cm}^{-2}$.

nature of the data, which would lead to unreliable values. However, the starting point of the noise can be taken as the frequency at which the electrochemical reaction begins to take place, and the nature of the chemical reactions has been reported elsewhere. ${ }^{14}$
Note that the high LF capacitance $\left(C_{1}\right)$ with values of millifarad has been observed under illumination and depends noticeably on the type of contact, and it has been associated with an electronic accumulation at the electrodes' interfaces. ${ }^{22}$ In the case of Au only, 
contact IF arc and the inductive loop indicates poor charge extraction capability, due to the lack of hole selective layer-metal oxide contact. Fitting results, without including the inductive elements, show a large $R_{\text {series }}$ for fresh and activated devices $\left(\sim 800 \Omega / \mathrm{cm}^{2}\right)$. Under light conditions, the nature of the resistances related to the arcs, $R_{3}$ and $R_{1}$, corresponds to losses from both bulk and interfacial recombination. ${ }^{19}$ High values of these resistances denote high resistance toward the recombination of carriers and better performance of the device. ${ }^{15}$ In our comparison, fresh samples show reduced recombination resistances in comparison to activated samples. These results are in agreement with improved photovoltage and photocurrent of the activated devices, as a consequence of reduced recombination kinetics.

\section{CONCLUSIONS}

This work establishes IS as a powerful tool to investigate chemical reactivity at the perovskite/external contact interface providing kinetic information. Selecting a system that shows chemical modifications in a timescale that can be analyzed by IS, ionic double layer generation is decoupled from chemical reactivity and electronic accumulation at the contacts. The current results open a new avenue to study the stability of perovskite solar cells under real working conditions focusing on the necessity to identify the chemical reactions that occurs at the perovskite/contacts interfaces.

\section{METHODS}

\section{Materials and precursor solutions}

All materials were used as received: FTO glasses $\left(25 \times 25 \mathrm{~mm}^{2}\right.$, Pilkington TEC15, $\sim 15 \Omega$ /sq resistance), $\mathrm{TiO}_{2}$ paste (Dyesol $30 \mathrm{NRD}$, $300 \mathrm{~nm}$ average particle size), $\mathrm{CH}_{3} \mathrm{NH}_{3} \mathrm{Br}$ (DYESOL), $\mathrm{PbBr}_{2}$ (TCI, 99.99\%), titanium diisopropoxide bis(acetylacetonate) (75\% in isopropanol, Sigma-Aldrich), and Spiro-OMeTAD (Merck). The $\mathrm{MAPbBr}_{3}$ perovskite precursor solution was prepared by dissolving the precursors separately to finally form a homogeneous solution of $1.4 \mathrm{M}$ concentration. The Spiro-OMeTAD solution for devices was prepared by dissolving in $1 \mathrm{ml}$ of chlorobenzene $72.3 \mathrm{mg}$ of $2,2^{\prime}, 7,7^{\prime}$-tetrakis $\left(N, N^{\prime}\right.$-di- $p$-methoxyphenylamine) $-9,9^{\prime}$ spirobifluorene, $28.8 \mu \mathrm{L}$ of 4 -tert-butylpyridine, and $17.5 \mu \mathrm{L}$ of a stock solution of $520 \mathrm{mg} / \mathrm{ml}$ of lithium bis-(trifluoromethylsulfonyl) imide in acetonitrile.

\section{Photovoltaic device fabrication}

All the processes were carried out inside the glovebox with oxygen and water conditions controlled. Photovoltaic devices were prepared over FTO glasses, which were partially etched with zinc powder and $\mathrm{HCl}(2 \mathrm{M})$. The substrates were cleaned with Hellmanex solution and rinsed with Milli-Q water and ethanol. Then, the substrates were sonicated for $15 \mathrm{~min}$ in a solution of acetone:isopropanol $(1: 1 \mathrm{v} / \mathrm{v})$ and dried. After that, the substrates were treated in a UV $-\mathrm{O}_{3}$ chamber for $15 \mathrm{~min}$. The $\mathrm{TiO}_{2}$ blocking layer was deposited onto the substrates by spray pyrolysis at $450{ }^{\circ} \mathrm{C}$ during $30 \mathrm{~min}$, using a titanium diisopropoxide bis(acetylacetonate) solution diluted in ethanol with oxygen as carrier gas. The mesoporous $\mathrm{TiO}_{2}$ layer was deposited by spin coating at $2000 \mathrm{rpm}$ during $10 \mathrm{~s}$ using a $\mathrm{TiO}_{2}$ paste diluted in ethanol. After drying at $100^{\circ} \mathrm{C}$ for $10 \mathrm{~min}$, the $\mathrm{TiO}_{2}$ mesoporous layer was heated at $500^{\circ} \mathrm{C}$ for $30 \mathrm{~min}$ and later cooled to room temperature. The perovskite precursor solution was spin-coated using toluene as antisolvent and heating up at $100^{\circ} \mathrm{C}$ for $3 \mathrm{~min}$. For HSL layer, a solution of Spiro-OMeTAD was prepared by dissolving $72.3 \mathrm{mg}$ of $2,2^{\prime}, 7,7^{\prime}$-tetrakis $\left(N, N^{\prime}\right.$-di-pmethoxyphenylamine)-9, $9^{\prime}$-spirobifluorene in $1 \mathrm{ml}$ of chlorobenzene, $28.8 \mu \mathrm{L}$ of 4 -tert-butylpyridine, and $17.5 \mu \mathrm{L}$ of a stock solution of $520 \mathrm{mg} / \mathrm{ml}$ of lithium bis-(trifluoromethylsulfonyl) imide in acetonitrile, as additives. Perovskite film was then covered when required with the HSL solution by dynamically spin coating at $4000 \mathrm{rpm}$ for $30 \mathrm{~s}$. Finally, $60 \mathrm{~nm}$ of gold was thermally evaporated on top of the device to form the metal electrode contacts using a commercial Univex 250 chamber, from Oerlikon Leybold Vacuum.

\section{Film and device characterization}

Scanning electron microscopy: The morphology of the films was observed using field-emission SEM using either a JEOL 7001F. SEM micrographs were further analyzed using the corresponding images. Photovoltaic devices were characterized using an Abett Solar simulator equipped with 1.5 AM filter. The light intensity was adjusted to $100 \mathrm{~mW} \mathrm{~cm}^{-2}$ using a calibrated Si solar cell. Devices were measured using a mask to define an active area of $0.11 \mathrm{~cm}^{2}$. Impedance spectroscopy measurements were performed using an Autolab PGSTAT-30 equipped with a frequency analyzer module. Measurements were carried out at different either in the dark or at 1 sun illumination conditions without using a masks to avoid creation of different areas that can contribute to the impedance of the sample. The DC bias was selected at either $0-0.2 \mathrm{~V}$ in the dark or adjusted to the measured $V_{o c}$ of the device under illumination conditions.

\section{Measurements protocol}

Due to the chemical reactivity of the interface and its kinetics, the sequence of the measurements is very important, and in the following discussion, the measurements start from small bias (voltage or light) and then move toward stronger bias. $J$ - $V$ curve is first measured at $500 \mathrm{mV} / \mathrm{s}$ in the dark and followed by 1 sun conditions. Then, IS is measured in the dark at $0 \mathrm{~V}$, followed by $0.2 \mathrm{~V}$, and finally under illumination at $V=V_{o c}$. Then, cyclic voltammetry measurement is carried out during a period of $20 \mathrm{~min}$ at varying scan rates to test the performance evolution. Measurements after 10 and $20 \mathrm{~min}$ are shown in the main text. Since the performance has improved over the time and the chemical reactivity has been promoted at this stage, the sample is so-called activated. Then, IS is measured in the dark at $0 \mathrm{~V}$ followed by measurement under illumination at 1 sun and $V=V_{o c}$.

\section{SUPPLEMENTARY MATERIAL}

See the supplementary material for further impedance spectra and IS fitting parameters. 


\section{ACKNOWLEDGMENTS}

We thank financial support from Ministerio de Ciencia, Innovación y Universidades of Spain, under Project (No. MAT201676892-C3-1-R). A.G. would like to thank MICINN for a Ramón y Cajal Fellowship (Grant No. RYC-201416809). University Jaume I is also acknowledged for financial support (Grant No. UJI-B2017-32). Serveis Centrals at UJI (SCIC) are acknowledged for the SEM measurement assistance.

\section{REFERENCES}

${ }^{1}$ Q. Jiang, Y. Zhao, X. Zhang, X. Yang, Y. Chen, Z. Chu, Q. Ye, X. Li, Z. Yin, and J. You, "Surface passivation of perovskite film for efficient solar cells," Nat. Photonics 13, 460 (2019).

${ }^{2}$ L. Zhang, X. Yang, Q. Jiang, P. Wang, Z. Yin, X. Zhang, H. Tan, Y. Yang, M. Wei, B. R. Sutherland, E. H. Sargent, and J. You, "Ultra-bright and highly efficient inorganic based perovskite light-emitting diodes," Nat. Commun. 8, 15640 (2017). ${ }^{3}$ Z. Lian, Q. Yan, Q. Lv, Y. Wang, L. Liu, L. Zhang, S. Pan, Q. Li, L. Wang, and J.-L. Sun, "High-performance planar-type photodetector on (100) facet of MAPbI3 single crystal," Sci. Rep. 5, 16563 (2015).

${ }^{4}$ W. Heiss and C. Brabec, "Perovskites target X-ray detection," Nat. Photonics 10, 288 (2016).

${ }^{5}$ A. Guerrero, J. You, C. Aranda, Y. S. Kang, G. Garcia-Belmonte, H. Zhou, J. Bisquert, and Y. Yang, "Interfacial degradation of planar lead halide perovskite solar cells,” ACS Nano 10, 218-224 (2016).

${ }^{6}$ H. Wang, A. Guerrero, A. Bou, A. M. AlMayouf, and J. Bisquert, "Kinetic and material properties of interfaces governing slow response and long timescale phenomena in perovskite solar cells," Energy Environ. Sci. 12, 2054 (2019).

${ }^{7}$ K. Domanski, J.-P. Correa-Baena, N. Mine, M. K. Nazeeruddin, A. Abate, M. Saliba, W. Tress, A. Hagfeldt, and M. Grätzel, "Not all that glitters is gold: Metal-migration-induced degradation in perovskite solar cells," ACS Nano 10, 6306-6314 (2016).

${ }^{8}$ M. Abdi-Jalebi, Z. Andaji-Garmaroudi, S. Cacovich, C. Stavrakas, B. Philippe, J. M. Richter, M. Alsari, E. P. Booker, E. M. Hutter, A. J. Pearson, S. Lilliu, T. J. Savenije, H. Rensmo, G. Divitini, C. Ducati, R. H. Friend, and S. D. Stranks, "Maximizing and stabilizing luminescence from halide perovskites with potassium passivation," Nature 555, 497 (2018).

${ }^{9}$ F. Fabregat-Santiago, J. Bisquert, G. Garcia-Belmonte, G. Boschloo, and A. Hagfeldt, "Influence of electrolyte in transport and recombination in dyesensitized solar cells studied by impedance spectroscopy," Sol. Energy Mater. Sol. Cells 87, 117-131 (2005).

${ }^{10}$ J. Song and M. Z. Bazant, "Effects of nanoparticle geometry and size distribution on diffusion impedance of battery electrodes," J. Electrochem. Soc. 160, A15-A24 (2013).

${ }^{11}$ A. Guerrero, G. Garcia-Belmonte, I. Mora-Sero, J. Bisquert, Y. S. Kang, T. J. Jacobsson, J.-P. Correa-Baena, and A. Hagfeldt, "Properties of contact and bulk impedances in hybrid lead halide perovskite solar cells including inductive loop elements," J. Phys. Chem. C 120, 8023-8032 (2016).

${ }^{12}$ A. Todinova, L. Contreras-Bernal, M. Salado, S. Ahmad, N. Morillo, J. Idígoras, and J. A. Anta, "Towards a universal approach for the analysis of impedance spectra of perovskite solar cells: Equivalent circuits and empirical analysis," ChemElectroChem 4, 2891-2901 (2017).
${ }^{13}$ W. Peng, C. Aranda, O. M. Bakr, G. Garcia-Belmonte, J. Bisquert, and A. Guerrero, "Quantification of ionic diffusion in lead halide perovskite single crystals," ACS Energy Lett. 3, 1477-1481 (2018).

${ }^{14}$ J. Pospisil, A. Guerrero, O. Zmeskal, M. Weiter, J. J. Gallardo, J. Navas, and G. Garcia-Belmonte, "Reversible formation of gold halides in single-crystal hybrid-perovskite/Au interface upon biasing and effect on electronic carrier injection," Adv. Funct. Mater. 29, 1900881 (2019).

${ }^{15}$ C. Aranda, A. Guerrero, and J. Bisquert, "Ionic effect enhances light emission and photovoltage of methylammonium lead bromide perovskite solar cell by reduced surface recombination,” ACS Energy Lett. 4, 741-746 (2019).

${ }^{16}$ S. Ravishankar, C. Aranda, S. Sanchez, J. Bisquert, M. Saliba, and G. GarciaBelmonte, "Perovskite solar cell modeling using light- and voltage-modulated techniques," J. Phys. Chem. C 123, 6444-6449 (2019).

${ }^{17}$ O. Almora, I. Zarazua, E. Mas-Marza, I. Mora-Sero, J. Bisquert, and G. GarciaBelmonte, "Capacitive dark currents, hysteresis, and electrode polarization in lead halide perovskite solar cells,” J. Phys. Chem. Lett. 6, 1645-1652 (2015).

${ }^{18}$ A. Guerrero, E. J. Juarez-Perez, J. Bisquert, I. Mora-Sero, and G. GarciaBelmonte, "Electrical field profile and doping in planar lead halide perovskite solar cells,” Appl. Phys. Lett. 105, 133902 (2014).

${ }^{19}$ J. Carrillo, A. Guerrero, S. Rahimnejad, O. Almora, I. Zarazua, E. Mas-Marza, J. Bisquert, and G. Garcia-Belmonte, "Ionic reactivity at contacts and aging of methylammonium lead triiodide perovskite solar cell," Adv. Energy Mater. 6, 1502246 (2016)

${ }^{20}$ O. Almora, A. Guerrero, and G. Garcia-Belmonte, "Ionic charging by local imbalance at interfaces in hybrid lead halide perovskites," Appl. Phys. Lett. 108, 043903 (2016).

${ }^{21}$ E. J. Juarez-Perez, R. S. Sanchez, L. Badia, G. Garcia-Belmonte, V. GonzalezPedro, Y. S. Kang, I. Mora-Sero, and J. Bisquert, "Photoinduced giant dielectric constant in lead halide perovskite solar cells," J. Phys. Chem. Lett. 5, 2390-2394 (2014).

${ }^{22}$ H.-S. Kim, I.-H. Jang, N. Ahn, M. Choi, A. Guerrero, J. Bisquert, and N.-G. Park, "Control of $I-V$ hysteresis in $\mathrm{CH}_{3} \mathrm{NH}_{3} \mathrm{PbI}_{3}$ perovskite solar cell," J. Phys. Chem. Lett. 6, 4633-4639 (2015).

${ }^{23}$ I. M. Hermes, Y. Hou, V. W. Bergmann, C. J. Brabec, and S. A. L. Weber, “The interplay of contact layers: How the electron transport layer influences interfacial recombination and hole extraction in perovskite solar cells," J. Phys. Chem. Lett. 9, 6249-6256 (2018).

${ }^{24}$ A. Guerrero, A. Bou, G. Matt, O. Almora, T. Heumüller, G. Garcia-Belmonte, J. Bisquert, Y. Hou, and C. Brabec, "Switching off hysteresis in perovskite solar cells by fine-tuning energy levels of extraction layers," Adv. Energy Mater. 8, 1703376 (2018)

${ }^{25}$ I. Zarazua, J. Bisquert, and G. Garcia-Belmonte, "Light-induced space-charge accumulation zone as photovoltaic mechanism in perovskite solar cells," J. Phys. Chem. Lett. 7, 525-528 (2016).

${ }^{26} \mathrm{~L}$. Bai, "AC impedance of faradaic reactions involving electrosorbed intermediates: Examination of conditions leading to pseudoinductive behavior represented in three-dimensional impedance spectroscopy diagrams," J. Electrochem. Soc. 138, 2897-2907 (1991).

${ }^{27}$ R. F. A. Jargelius-Pettersson, "Examination of the role of molybdenum in passivation of stainless steels using AC impedance spectroscopy," J. Electrochem. Soc. 145, 1462-1469 (1998).

${ }^{28} \mathrm{C}$. Li, A. Guerrero, S. Huettner, and J. Bisquert, "Unravelling the role of vacancies in lead halide perovskite through electrical switching of photoluminescence," Nat. Commun. 9, 5113 (2018). 